\title{
Anticoagulants and Antithrombotics
}

\author{
Justyna Domienik-Karlowicz¹, Yuri Lopatin ${ }^{2}$
}

1. Department of General Medicine and Cardiology, Medical University of Warsaw, Lindley'a 5, 02-005 Warsaw, Poland

2. Professor and Head of Cardiology, Volgograd Regional Cardiology Centre, Volgograd, Russia

\section{Corresponding author:}

Yuri Lopatin, Professor and Head of Cardiology,

Volgograd Regional Cardiology Centre, Volgograd, Russia

Email: yu.lopatin@gmail.com

\begin{abstract}
Hospitalized and stable HF patients are both at increased risk of thrombo-embolic events, making the possibility of protection via the use of anticoagulants and/or antithrombotics attractive. The 2016 ESC guidelines on HF, do not recommend aspirin or anticoagulation in HF patients in SR, or any antiplatelet drugs in patients with HF without concomitant CAD. NOACs have a favourable safety profile and simplicity of use. This has led to interest in their use in HF. Secondary analyses showed that patients with HF with high cardiovascular risk and/or CAD may benefit from NOACs. However, these benefits in HF patients in SR were not confirmed in the large COMMANDER HF trial where rivaroxaban $2.5 \mathrm{mg}$ twice daily did not reduce the primary endpoint (death from any cause, MI, stroke), nor achieve its primary safety outcome. In patients with HF in AF guidelines recommend an oral anticoagulant to prevent thrombo-embolism for all patients with paroxysmal or persistent/permanent AF and CHA2DS2 VASc $\geq 2$, and for patients with HF and non-valvular AF, eligible for anticoagulation based on a CHA2DS2-VASc score, NOACs rather than warfarin should be considered.
\end{abstract}

Keywords: heart failure; anticoagulant; anti-thrombotic

Citation: Domienik-Karlowicz J, Lopatin Y. Anticoagulants and Antithrombotics. International Cardiovascular Forum Journal. 2019;18:23-26. DOI: 10.17987/icfj.v18i0.619

\section{Introduction}

A substantial number of studies have provided evidence of the link between thrombosis and heart failure (HF). It is important to understand that thrombosis can be both a cause and a consequence of HF. HF itself promotes a state of hypercoagulability, and HF, its complications and progression may result from the consequences of a pre-existing prothrombotic state. Many HF patients routinely receive anticoagulant or antithrombotic therapy due to the presence of specific indications for their use, such as atrial fibrillation (AF) or coronary artery disease (CAD). However, the potential clinical benefit of using these agents in the management of HF itself remains a subject of active discussions.

\section{Potential pathophysiological mechanisms leading to thrombosis in heart failure}

HF interferes with 3 elements included in the Virchow triad, leading to:

- hypercoagulability, due to increased plasma viscosity, platelet activation, impairment of the protein $\mathrm{C}$ pathway and thrombin formation, adenosine-mediated thrombosis;

- haemodynamic changes (stasis) due to low cardiac output, cardiac chamber dilatation, aneurysms of the left ventricle (LV), impaired myocardial contractility;

- endothelial injury/dysfunction related to oxidative stress, a pro-inflammatory state, deficient NO production and many others.

\section{Epidemiology of thrombotic events in patients with heart failure}

Pulmonary embolism, peripheral embolism, stroke.

Data from observational studies and secondary analyses of randomized clinical trials indicate a higher risk of thrombotic events in patients with HF than in general population.[1] It was found that in hospitalized HF patients the relative risk for pulmonary embolism and venous thromboembolism (VTE) was 2.15 and 1.21, respectively.[2] Interestingly, the NT-proBNP level may be more useful than the NYHA class as an indicator for identifying patients at a high short-term risk of VTE, whereas elevated D-dimer may be suggestive of high mid-term risk.[3] In patients with HFrEF and in sinus rhythm (SOLVD study), the annual incidence of stroke, pulmonary and peripheral embolism was 2.4 $\%$ in women and $1.8 \%$ in men. Moreover, a $53 \%$ increased risk of VTE was observed for every $10 \%$ of reduction in LVEF. [4] Tang et al. in their meta-analysis of 71 studies investigating the risk of VTE in patients hospitalized due to HF found that the overall median symptomatic VTE rate was $2.48 \%$.[5] However, for patients who did not receive thromboprophylaxis the VTE rate was already $3.73 \%$ compared to $1.47 \%$ for those who did.

In the Framingham Study based on 5,184 participants over 24 years, the adjusted risk ratio for stroke was 5.4 in men and 6.2 in women (with no adjustment for a presence of AF).[6] Moreover, in a 2007 meta-analysis, 18 in every 1,000 persons with HF 
experienced a stroke during the first year after the diagnosis of HF, with a maximum stroke rate of 47.4 for every 1000 patients observed after 5-years of follow-up.[7] In the Rotterdam Study, based on 7,546 patients with no history of stroke, the risk of ischaemic stroke increased almost 6-fold during the first month after the diagnosis of HF, but decreased to 3.5 fold for 1-6 months post-onset, and was not significantly elevated from 6 months onwards.[8]

LV thrombi. Epidemiological data on the risk of thromboembolism in patients with LV thrombi and HF are unavailable. Contemporary data estimate the prevalence of LV thrombus at about 15\% in patients with ST-segment elevation myocardial infarction and $25 \%$ in patients with anterior myocardial infarction. [8]

\section{Clinical models estimating the thromboembolic risk in patients with heart failure}

Data from the CORONA and GISSI-HF trials were re-analyzed in order to estimate the actual incidence of, and risk factors for, stroke in patients with HF without AF.[10] Two clinical models for stroke risk in patients, with $\mathrm{HF}$ and without AF, were developed. The first model included the following clinical variables: NYHA class, age, insulin-dependent type 2 diabetes mellitus, and the history of previous stroke and body mass index. The second model included plasma NT pro-BNP, type 2 diabetes mellitus, and the history of previous stroke. Interestingly, patients with $\mathrm{HF}$ without $\mathrm{AF}$ in the upper tercile of the risk score had a rate of stroke that approximated to the rate of risk in HF patients with AF. Both models will require further validation, before they can be recommended for a broader clinical use.

In another study, the predictive accuracy of the CHA2DS2-VASc score was validated in a population of patients with HF $(42,987$ patients from Danish registries not receiving anticoagulation).[11] The absolute risk of thromboembolism was high independently of the presence of AF, beginning from the CHA2DS2-VASc score exceeding 4. Importantly, the risk was greater with an increasing CHA2DS2-VASc score, but the predictive accuracy was only modest. The clinical utility of the CHA2DS2-VASc score in patients with $\mathrm{HF}$ without $\mathrm{AF}$ remains to be determined.

\section{Data on safety and efficacy of antithrombotic/ anticoagulants therapy in patients with heart failure Patients with heart failure in sinus rhythm.}

According to the 2016 ESC guidelines on HF [12], in patients with $\mathrm{HF}$ who are in sinus rhythm, there is no evidence that oral anticoagulants decrease mortality and morbidity in comparison to placebo or aspirin. Moreover, no benefit has been observed when using antiplatelet drugs in patients with HF without concomitant CAD. WARCEF investigators [13] demonstrated no significant overall difference between warfarin and aspirin in preventing the primary outcome defined as a time to first event in a composite endpoint of ischemic stroke, intracerebral hemorrhage, or death from any cause. Along with that, warfarin therapy was associated with a significant reduction in the risk of stroke compared to aspirin without a significant difference in the risk of intracranial and intracerebral hemorrhages. However, the rate of major hemorrhage was significantly higher with warfarin compared to aspirin, largely due to a more frequent occurrence of major gastrointestinal bleeding.
The advent of NOACs, agents with a favorable safety profile and simplicity of use, has led to further attempts to use them in HF patients. There were some suggestions from secondary analyses that patients with HF with high cardiovascular risk and/or coronary artery disease may benefit from the addition of NOAC therapy. A subgroup analysis from the ATLAS ACS 2-TIMI 51 trial (patients with a recent ACS on dual antiplatelet therapy) showed that rivaroxaban $2.5 \mathrm{mg}$ daily compared with placebo reduced the primary outcome of cardiovascular death, myocardial infarction or stroke, and all-cause death in patients having HF at the time of their ACS.[14] Also a sub-analysis of patients with HF enrolled in the COMPASS trial (patients with a history of stable atherosclerotic disease) suggested some benefits from rivaroxaban treatment ( $2.5 \mathrm{mg}$ twice daily) in addition to aspirin (100 mg daily).[14]

Both above-mentioned studies noted that the beneficial effects of rivaroxaban were accompanied by an increased rate of bleeding.[14, 15] However, the expectation of additional benefits of including rivaroxaban to standard care in patients HF who are in sinus rhythm has not been confirmed in the COMMANDER HF trial.[16] This double-blind, randomized controlled trial was designed to assess the effectiveness and safety of rivaroxaban in reducing thrombin generation, and as a consequence, mortality, myocardial infarction, or stroke in patients with worsening chronic HF, reduced LVEF, CAD, and no AF. It was shown that rivaroxaban treatment at a dose of $2.5 \mathrm{mg}$ twice daily was not associated with a significantly lower risk of the primary endpoint (death from any cause, myocardial infarction, stroke), nor the primary safety outcome (fatal bleeding or bleeding into a critical space with a potential for causing permanent disability). Moreover, rivaroxaban did not affect the rate of rehospitalization for worsening HF. Thus, the results of the COMMANDER HF trial [16] did not confirm the contribution of atherothrombotic coronary events to the progression of HFrEF of ischaemic aetiology.

\section{Patients with heart failure and atrial fibrillation.}

The 2016 ESC guidelines on HF emphasize that patients with $\mathrm{HF}$ and AF should generally be anticoagulated, therefore an evaluation of the balance of benefit and the risk of bleeding (using CHA2DS2-VASc and HAS-BLED scores) is indicated. [12] A substantial proportion of patients with $H F$ and $A F$ is characterized by both benefit and risk scores $\geq 3$, indicating that careful consideration should be given before prescribing an oral anticoagulant and that regular patient's control is subsequently needed.

The 2016 ESC guidelines on HF make the following recommendations for the prevention of thromboembolism in patients with $\mathrm{HF}$ and concurrent $\mathrm{AF}$ [12]:

1. For the estimation of the risk of thromboembolism and the risk of bleeding associated with oral anticoagulation, the $\mathrm{CHA}_{2} \mathrm{DS}_{2}-$ VASc and HAD-BLED scores are recommended tools (IB)

2. An oral anticoagulant is recommended to prevent thromboembolism for all patients with paroxysmal or persistent/permanent $\mathrm{AF}$ and $\mathrm{CHA}_{2} \mathrm{DS}_{2}-\mathrm{VASc} \geq 2$ without contraindications, and irrespective of whether a rate or rhythm management strategy is used (including after successful cardioversion) (IA)

3. NOAC treatment is contraindicated in patients with mechanical valves, or at least moderate mitral stenosis (IIIB) 
Table 1. The hazard ratio (HR) and $95 \%$ confidence interval of the efficacy and safety outcomes of NOACs compared with warfarin in AF patients with or without HF

\begin{tabular}{|c|c|c|c|c|c|}
\hline Clinical Trial & \multicolumn{2}{|c|}{ RE-LY [17] } & ROCKET-AF [18] & ARISTOTLE [19] & $\begin{array}{l}\text { ENGAGE } \\
\text { AF-TIMI } 46 \text { [20] }\end{array}$ \\
\hline \multirow[t]{2}{*}{ NOAC } & \multicolumn{2}{|c|}{ Dabigatran } & \multirow[t]{2}{*}{ Rivaroxaban } & \multirow[t]{2}{*}{ Apixaban } & \multirow[t]{2}{*}{ Edoxaban } \\
\hline & $110 \mathrm{mg}$ & $150 \mathrm{mg}$ & & & \\
\hline $\begin{array}{l}\text { AF patients } \\
\text { with HF, n }\end{array}$ & 1641 & 1640 & 4530 & 3235 & 8145 \\
\hline $\begin{array}{l}\text { Stroke/systemic } \\
\text { embolism }\end{array}$ & $0.99(0.69-1.42)$ & $0.75(0.51-1.10)$ & $0.91(0.74-1.13)$ & $\begin{array}{l}0.55(0.34-0.91) \dagger \\
0.98(0.65-1.49) \ddagger\end{array}$ & $\begin{array}{l}0.88(0.69-1.12)^{+} \\
0.83(0.55-1.25)^{++}\end{array}$ \\
\hline Major bleeding & $0.83(0.64-1.09)$ & $0.79(0.60-1.03)$ & N/A & $\begin{array}{l}0.81(0.58-1.14) \dagger 0.62 \\
(0.44-0.88) \ddagger\end{array}$ & $\begin{array}{l}0.79(0.65-0.96)^{+} \\
0.79(0.54-1.17)^{++}\end{array}$ \\
\hline $\begin{array}{l}\text { Intracranial } \\
\text { haemorrhage }\end{array}$ & $0.34(0.14-0.80)$ & $0.39(0.17-0.89)$ & $0.63(0.40-1.02)$ & $0.25(0.08-0.73) \dagger 0.20(0.07-0.58) \ddagger$ & $\begin{array}{l}0.45(0.28-0.73)^{+} \\
0.35(0.14-0.88)^{++}\end{array}$ \\
\hline $\begin{array}{l}\text { AF patients } \\
\text { without HF, n }\end{array}$ & 4374 & 4436 & 2551 & 5885 & 5926 \\
\hline $\begin{array}{l}\text { Stroke/systemic } \\
\text { embolism }\end{array}$ & $0.86(0.67-1.09)$ & $0.61(0.47-0.79)$ & $0.84(0.65-1.09)$ & $0.74(0.57-0.96)$ & $0.87(0.69-1.11)$ \\
\hline Major bleeding & $0.79(0.67-0.94)$ & $0.99(0.84-1.16)$ & N/A & $0.77(0.62-0.94)$ & $0.82(0.68-0.99)$ \\
\hline $\begin{array}{l}\text { Intracranial } \\
\text { haemorrhage }\end{array}$ & $0.28(0.17-0.47)$ & $0.42(0.27-0.64)$ & 1.05 (0.93-1.18) & $0.47(0.30-0.73)$ & $0.51(0.33-0.80)$ \\
\hline \multicolumn{6}{|c|}{$\begin{array}{l}\text { Legend: } \\
\text { N/A, not available } \\
\text { †HR and } 95 \% \mathrm{Cl} \text { for patients with left ventricular systolic dysfunction. } \\
\text { †HR and } 95 \% \mathrm{Cl} \text { for patients with heart failure and preserved ejection fraction. } \\
{ }^{+} \mathrm{HR} \text { and } 95 \% \mathrm{Cl} \text { for patients with heart failure NYHA I-II } \\
{ }^{++} \mathrm{HR} \text { and } 95 \% \mathrm{Cl} \text { for patients with heart failure NYHA III-IV }\end{array}$} \\
\hline
\end{tabular}

4. Combination of an oral anticoagulant and an antiplatelet agent is not recommended in patients with chronic (>12 months after an acute event) coronary or other arterial disease, because of a high-risk of serious bleeding. Single therapy with an oral anticoagulant is preferred after 12 months (IIIC)

5. For patients with $\mathrm{HF}$ and non-valvular $\mathrm{AF}$, eligible for anticoagulation based on a $\mathrm{CHA}_{2} \mathrm{DS}_{2}$-VASc score, NOACs rather than warfarin should be considered for anticoagulation as NOACs are associated with a lower risk of stroke, intracranial hemorrhage and mortality, which outweigh the increased risk of gastrointestinal haemorrhage (IlaB)

Data on efficacy and safety of NOACs (dabigatran, rivaroxaban, apixaban and edoxaban) compared with warfin in additional analyses in patients with AF with HF versus without HF are summarized in table 1 . Based on the results of the metaanalysis [21], which included patients with AF and HF (13,384 patients treated with NOACs and 13390 patients treated with warfarin), single-/high-dose NOAC regimens have a significantly better efficacy and safety profile compared with warfarin. Lowdose regimens had similar efficacy and safety levels to those of warfarin. Currently, head-to-head comparisons between NOACs in patients with AF and HF are not available.

\section{Patients with LV.}

According to the ESC guidelines for the management of acute $\mathrm{MI}$ in patients presenting with ST-segment elevation, when LV thrombus is recognized, anticoagulation should be administered for up to 6 months guided by repeated imaging.[22] The standard care for patients with LV thrombus is anticoagulation with warfarin, this practice is based on studies performed in the thrombolytic era in post MI patients. The results of the use of NOACs in patients with LV thrombus were combined in a meta-summary of 36 clinical cases, where HF was one of the most common comorbidities [23]. LV thrombus resolution with NOACs was noted in most patients with median duration of treatment to resolution equal to 1 month. There were minimal bleeding and no embolic events reported. Further clinical trials will provide more robust data regarding the efficacy and safety of NOACs in patients with LV thrombus.

\section{Conclusions}

To this date, there still is no conclusive evidence that HF itself should be an indication for anticoagulant/antithrombotic therapy. Recommendations for anticoagulant therapy in AF are similar in patients, with and without HF. The efficacy and safety of NOACs are similar, regardless of concomitant HF.

\section{Declarations of interest}

The authors declare no conflict of interest.

\section{Acknowledgements}

The authors state that they abide by the authors' responsibilities and ethical publishing guidelines of the International Cardiovascular Forum Journal.[24]

\section{References}

1. Bettari, L., et al., Thromboembolism and antithrombotic therapy in patients with heart failure in sinus rhythm: current status and future directions. Circ Heart Fail, 2011. 4(3): p. 361-8.

2. Beemath A, Stein PD, Skaf E, Al Sibae MR, et al. Risk of venous thromboembolism in patients hospitalized with heart failure. Am J Cardiol. 2006; 98 (6): 793-795.

3. Mebazaa A, et al. Predicting the risk of venous thromboembolism in patients hospitalized with heart failure. Circulation. 2014; 130(5): 410-418. 
4. Al-Khadra AS, et al. Antiplatelet agents and survival: a cohort analysis from the Studies of Left Ventricular Dysfunction (SOLVD) trial. J Am Coll Cardiol, 1998; 31(2): 419-425.

5. Tang, L., et al., Heart failure and risk of venous thromboembolism: a systematic review and meta-analysis. Lancet Haematol, 2016. 3(1): p. e30-44.

6. Kannel, W.B., P.A. Wolf, and J. Verter, Manifestations of coronary disease predisposing to stroke. The Framingham study. JAMA, 1983. 250(21): p. 2942-6.

7. Witt, B. et al., The incidence of ischemic stroke in chronic heart failure. JCF 2007.13:489.

8. Alberts, V.P., et al., Heart failure and the risk of stroke: the Rotterdam Study. Eur J Epidemiol, 2010. 25(11): p. 807-12.

9. McCarthy, C.P., et al., Left Ventricular Thrombus After Acute Myocardial Infarction: Screening, Prevention, and Treatment. JAMA Cardiol, 2018. 3(7): p. 642-649.

10. Abdul-Rahim, A.H., et al., Risk of Stroke in Chronic Heart Failure Patients Without Atrial Fibrillation: Analysis of the GISSI-HF Trials. Circ, 2015. 131:1486-94.

11. Melgaard, L., et al., Assessment of the CHA2DS2-VASc Score in Predicting Ischemic Stroke, Thromboembolism, and Death in Patients With Heart Failure With and Without Atrial Fibrillation. JAMA, 2015. 314(10): p. 1030-8.

12. Ponikowski P, et al. 2016 ESC Guidelines for the diagnosis and treatment of acute and chronic heart failure. Eur Heart J. 2016; 37(27): 2129-2200.

13. Homma, S., et al., Warfarin and aspirin in patients with heart failure and sinus rhythm. N Engl J Med, 2012. 366(20): p. 1859-69.

14. Mega, J.L., et al., Rivaroxaban in patients with a recent acute coronary syndrome. N Engl J Med, 2012. 366(1): p. 9-19.

15. Connolly, S.J., Eikelboom JW, Bosch J, et al., Rivaroxaban with or without aspirin in patients with stable coronary artery disease. Lancet, 2017.39:205218.

16. Zannad, F., et al., Rivaroxaban in Patients with Heart Failure, Sinus Rhythm, and Coronary Disease. N Engl J Med, 2018. Oct 4;379(14):1332-1342.

17. Ferreira J, Ezekowitz MD, Connolly SJ, Brueckmann M, et al. Dabigatran compared with warfarin in patients with atrial fibrillation and symptomatic heart failure: a subgroup analysis of the RE-LY trial. Eur J Heart Fail 2013; 15 (9): 1053-1061.

18. van Diepen S, et al. Efficacy and safety of rivaroxaban in patients with heart failure and nonvalvular atrial fibrillation: insights from ROCKET AF. Circ Heart Fail 2013;6:740-7

19. McMurray JJ, Ezekowitz JA, Lewis BS, Gersh BJ, et al. Left ventricular systolic dysfunction, heart failure, and the risk of stroke and systemic embolism in patients with atrial fibrillation: insights from the ARISTOTLE trial. Circ Heart Fail 2013; 6 (3): 451-460

20. Magnani G, Giugliano RP, Ruff CT, Murphy SA, et al. Efficacy and safety of edoxaban compared with warfarin in patients with atrial fibrillation and heart failure: insights from ENGAGE AF-TIMI 48. Eur J Heart Fail. 2016; 18(9): 1153-1161.

21. Xiong, Q., et al., Non-vitamin K antagonist oral anticoagulants (NOACs) in patients with concomitant atrial fibrillation and heart failure: a systemic review and meta-analysis of randomized trials. Eur J Heart Fail, 2015. 17(11): p. 1192-1200

22. Ibanez B, et al. 2017 ESC Guidelines for the management of acute myocardial infarction in patients presenting with ST-segment elevation. EHJ. 2017;39: 119-177.

23. Leow AS, Sia CH, Tan BY, Loh JP. A meta-summary of case reports of nonvitamin $\mathrm{K}$ antagonist oral anticoagulant use in patients with left ventricular thrombus. J Thromb Thrombolysis. 2018; 46(1): 68-73.

24. Shewan LG, Coats AJS, Henein MY Authors' Responsibilities and Ethical Publishing. International Cardiovascular Forum Journal 2018;13:3-4, DOI: 10.17987/icfj.v13i0.525 